\title{
The Coherent Signals Processing Method in the Multiradar System of the Same Type Two-coordinate Surveillance Radars with Mechanical Azimuthal Rotation
}

\author{
Hennadii Khudov ${ }^{1}$, Vitaliy Lishchenko ${ }^{2}$, Boris Lanetskii ${ }^{3}$, \\ Vadym Lukianchuk ${ }^{4}$, Stanislav Stetsiv ${ }^{5}$, Ivan Kravchenko ${ }^{6}$ \\ ${ }^{1}$ Department of Radar Troops Tactic, Ivan Kozhedub Kharkiv National Air Force University, Kharkiv, Ukraine, \\ 2345kh_hg@ukr.net \\ ${ }^{2}$ Department of Radar Troops Tactic, Ivan Kozhedub Kharkiv National Air Force University, Kharkiv, Ukraine, \\ lvnpo4ta@gmail.com \\ ${ }^{3}$ Scientific center of Air Force, Ivan Kozhedub Kharkiv National Air Force University, Kharkiv, Ukraine, \\ Laneckij.B.hnups@gmail.com \\ ${ }^{4}$ Scientific center of Air Force, Ivan Kozhedub Kharkiv National Air Force University, Kharkiv, Ukraine, \\ super.vadim1973@ukr.net \\ ${ }^{5}$ Department of Missile Forces, Hetman Petro Sahaidachnyi National Army Academy, Lviv, Ukraine, \\ 2345kh_hg@ukr.net \\ ${ }^{6}$ Data-Processing Computer Centre, Ivan Kozhedub Kharkiv National Air Force University, Kharkiv, \\ Ukraine, kravchenkoivan@ukr.net
}

\begin{abstract}
The subject of the article is the task of developing a coherent signals processing method in the multiradar system of the same type two-coordinate surveillance radars with mechanical azimuthal rotation. The goal of the paper is solving the problem of developing a coherent signals processing method in multiradar system based on radars with mechanical rotation. It has been found that, in modern-day hybrid wars, founding a reliable air defence system is made much more difficult by the appearance of low radar cross section air objects. It is proposed to increase the energy potential of existing surveillance radars by integrating them into a spatially spaced coherent multiradar system. To ensure a consistent overview of the airspace, the locations of the radars are made in a single line with their synchronous azimuthal rotation. The transmitting signals must be simultaneously transmitted and orthogonal by frequency diversity or encoding. The synthesis of the optimal coherent signal finder is performed. The characteristics of detection of air objects in a multiradar system with mutually coherent signals are evaluated, when all radars emit identical signals in such a way that their common-purpose summation is secured. The following results were obtained: the highest efficiency in signal/noise gain was shown by the addition of a second radar, the optimum number of radars in the multi-radar system of no more than four. Therefore, the most cost-effective criterion is to create a spatially coherent multiradar system by combining two to four similar radars. In this case, the expected gain in the threshold signal-to-noise can be up to eighteen decibels. The use of more than four radars is impractical because it does not lead to a significant increase in the gain. The direction of
\end{abstract}

further researches is the development of a non-coherent mutually processing signals method in a multiradar system-space spatially spaced two-coordinate radar observation stations with mechanical azimuthal rotation.

Key words: radar, multiradar system, air object, coherent processing, detection curve, probability of correct detection, probability of false alarm.

\section{INTRODUCTION}

In the context of modern hybrid and network-centric wars, the anti-terrorist operation and the Joint Forces operation in eastern Ukraine, the construction of a reliable air defence system in general and its reconnaissance information subsystem is significantly complicated by the appearance of small visibility (with low radar contrast) ) and small air objects [1]-[8].

It is known from the experience of last local wars and the anti-terrorist operation and the Joint Forces operation that small UAVs in most cases were not detected by the available means of radar of the radar troops [2]-[5], [9], which form the basis of the air defence information subsystem.

The following problems significantly reduce the effectiveness of the using of radar troops [10]-[16]:

- the radar air surveillance system is not able to provide guaranteed detection and maintenance of the air object in general and inconspicuous objects in particular;

- deficiency of sufficient number of new and modernized samples of electronic equipment, means of automation of collection, processing and issuance of radar information in radio engineering units to provide information about the air object at low and extremely low altitudes, and others. 
World and domestic manufacturers of radar equipment implement the latest advances in science and technology: sequential-parallel electronic survey of the area around the corner and two-dimensional electronic scanning of the antenna pattern, adaptive phased array antennas, digital synthesis of transmitting signals with different parameters diagram formation [17]-[18].

Currently, the radar troops of the Air Force of the Armed Forces of Ukraine receive the latest and modernized models of radar, including broadband phase-locked signals and digital, but this process is gradual and expensive. Therefore, in general, the available equipment of the units does not fully solve the tasks assigned to the units, especially in the part related to the detection of inconspicuous air object.

At the same time, the radio engineering units are armed with a large number of two-coordinate survey radars of the "old" P-18 fleet. These radars are not able to detect modern inconspicuous air object with the specified quality indicators [2]-[3], [5].

Thus, at present, in solving the problem of detecting inconspicuous air object, the discrepancy between the development trends of the air object and the capabilities of existing radars to detect inconspicuous air object with the required efficiency has sharpened.

To address this discrepancy and increase the efficiency of detection of inconspicuous air objects, it is proposed to combine the existing survey two-coordinate radar into a coherent multiradar system with compatible signal processing [10], [12], [19]-[20].

The purpose of the article is developing the coherent signals processing method in a multi-radar system of the same type of two-coordinate survey radars with mechanical rotation.

\section{PROBLEM ANALYSIS}

On date, the tasks of aerial surveillance and detection of small radar by the reflection surface of an air object are solved by conducting surveillance, using radio and infrared, optical and acoustic means of reconnaissance. However, the use of radar reconnaissance has its undeniable advantages over the above methods [21]. In addition to the known methods of radar in recent years are developing and widely used non-traditional: the using of external backlight sources, using the effect of "radar on the lumen", the use of passive-active radar systems and others [21].

These methods are based on a general trend in the development of technology - the integration of individual devices (tools) into complex structured systems. It is known that there are a number of different types of multi-radar systems. The individual positions of the elements of such systems are spatially spaced from each other, while the processing of radar information is performed in the central processing point, which can be combined with one of the positions or located separately. At this point, it is advisable to carry out joint processing of radar information, the effectiveness of which depends on the degree of coherence provided in the system [19]. In this paper, it is proposed to combine the existing two-coordinate survey radars into a synchronous coherent multiradar system according to the method described in [19] and explained by the algorithm shown on Figure 1.

\section{Main Material}

Creating a multi-radar system makes it possible to solve the problem of increasing the energy potential of radar, increasing the gain of antenna systems or the use of system effects. The using of system effects when combining autonomous radars in a multi-radar system is associated with the possibility of implementing different degrees of coherence of spatially spaced positions and the combined reception of echoes [19]-[20]. One of the key issues that determine the possibility of creating a synchronous-coherent multi-radar system from spatially spaced radar is the need to accord the conditions [19]-[20]:

- frequency synchronization;

- phase synchronization;

- time synchronization;

- agreed space overview.

In Figure 2 shows an example of building a multi-radar system. Each of radars emits its own transmitting signal, which is not correlated with the transmitting signal of other radars. The signals of all radars are emitted simultaneously. Non-correlation (orthogonality) can be provided by:

- frequency diversity (frequency);

- coding of signals (code).

Each of radars receives echo signals:

- its transmitting signal reflected from the air object;

- the probing signal of neighbouring radars reflected from the air object.

Consider a multi-radar system, in which each of radars emits mutually orthogonal signals. These signals are reflected from an air object of complex shape. If the size of the air object is much larger than the wavelength, the signals received by each of radars fluctuate. As a rule, amplitude fluctuations are described by Rayleigh law, and phase fluctuations are evenly distributed in the interval $(-\pi, \pi)$. The character of the fluctuations of the signals received by different radars depends on the degree of their spatial correlation, which is determined by the size of the effective base (distance) between the radar (expression (1)):

$$
\frac{\mathrm{L}_{\mathrm{ef}}}{\mathrm{R}}>(0.8 \ldots 1) \frac{\lambda}{1_{\mathrm{AO}}},
$$


where $\mathrm{L}_{\mathrm{ef}}=\mathrm{L} \sin (\theta)$ - effective base between spatially spaced i-radar and j-radar $(\theta-$ is the angle that determines the direction of the air object);

$\mathrm{R}$ - distance to the air object from the middle of the effective base;

$\lambda$ - radar wavelength;

$1_{\mathrm{AO}}-$ transverse size of the air object (parallel to the effective base).

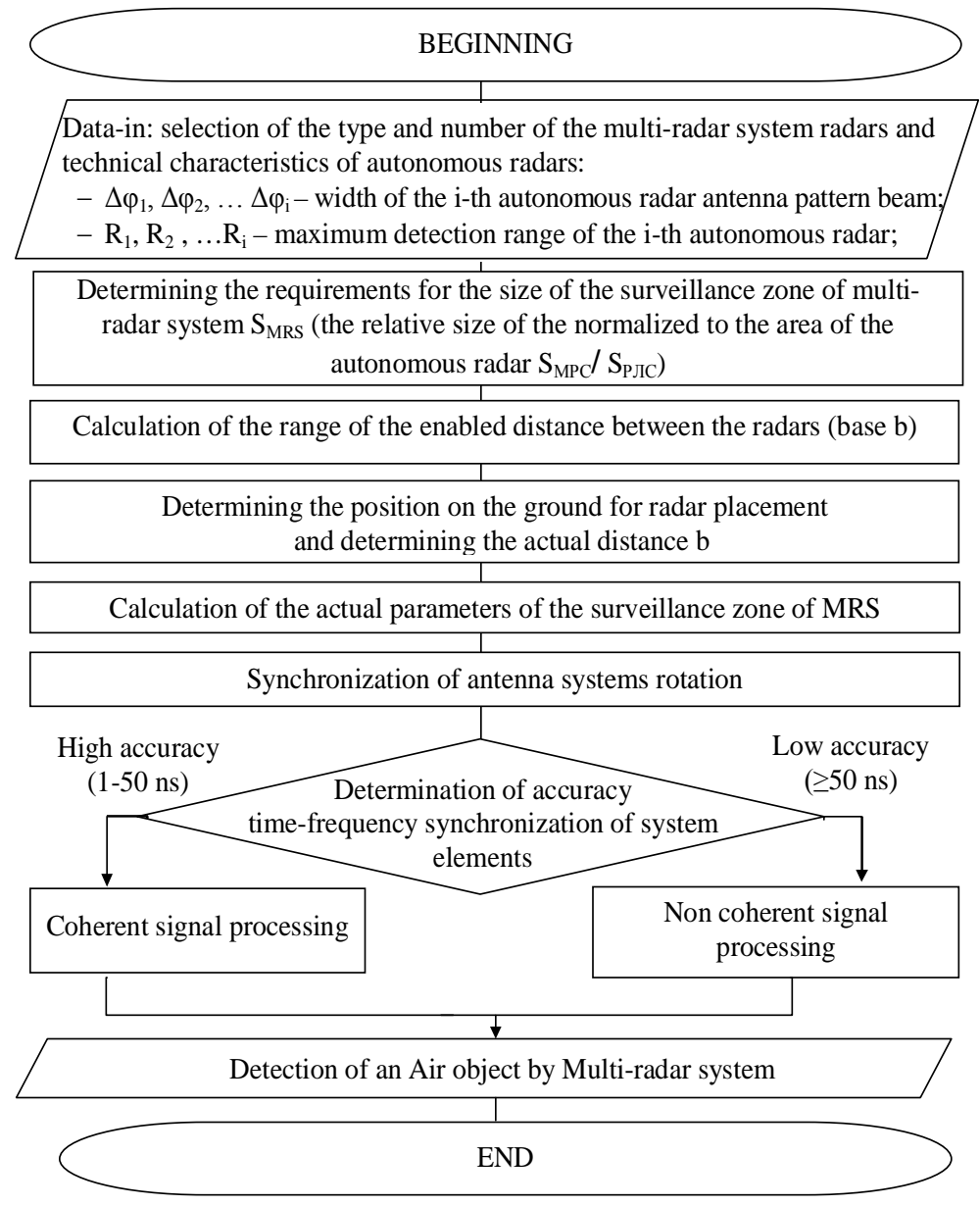

Figure 1: Algorithm that implements the method of forming a coordinated field of view of small base multi-radar system of the same type of radar with mechanical rotation

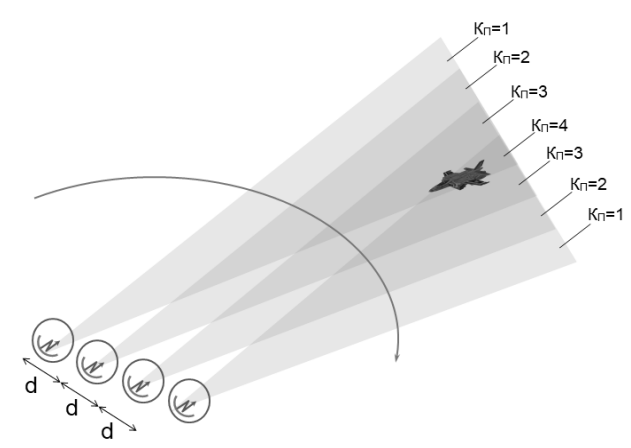

Figure 2: An example of building a synchronous small base multi-radar system with 4 radars

At full spatial correlation, the complex amplitudes and initial phases of the signals at the inputs of different positions are strongly connected and fluctuate amicably. Therefore, such signals are spatially coherent. At small bases between receiving positions rigid communication between complex amplitudes of echo signals in various positions (full time correlation) is possible.

The input of the radar receiver in each position receives signals emitted by all radars.

If all radars emit the same signals in such a way that they are in-phase summation on the air object, then there will be only one echo signal at the input of the receiving positions. The effective value of the amplitude of the echo signal in this case will be equal to the sum of the effective values of the echo signals formed by all transmitting positions [19]-[21].

In this case, the processing is performed on $\mathrm{M}$ implementations of independent Gaussian processes. The average value of these processes in each receiving position is equal to the phased sum of $\mathrm{M}$ echo signals. 
The initial value of the signal-to-noise is determined by expression (2):

$$
\mathrm{q}_{\text {exit }}^{2}=\mathrm{NM}^{2} \mathrm{q}_{\text {exit } 0}^{2}
$$

where $\mathrm{q}_{\text {exit }}^{2}$ - a signal-to-noise ratio at the output of a mono position radar;

$\mathrm{N}$ - the number of radars operating in transceiver mode; $\mathrm{M}$-implementations of independent Gaussian processes.
The additional gain in $\mathrm{M}$ times is explained by the fact that at in-phase addition of signals on air object noises of the receiver do not take part, and at coherent addition in receiving devices together with signals noise is incoherently.

The coherent signals method processing in a multi-radar system of the same type of two-coordinate survey radars with mechanical rotation is proposed in the form presented in Figure 3. Figure 4 shows the detection curves for a small base spatially coherent multi-radar system

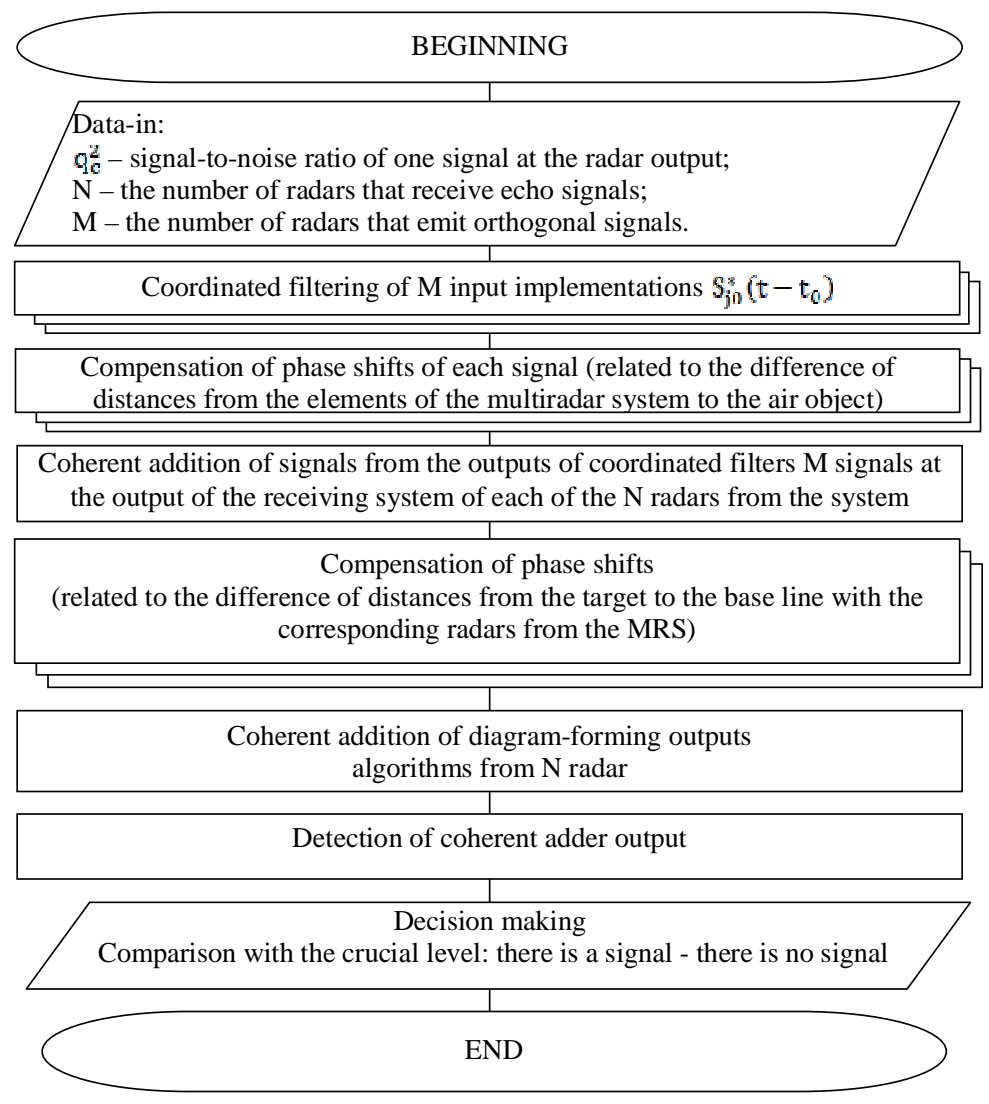

Figure 3: The algorithm that implements the coherent signals method processing in a multi-radar system

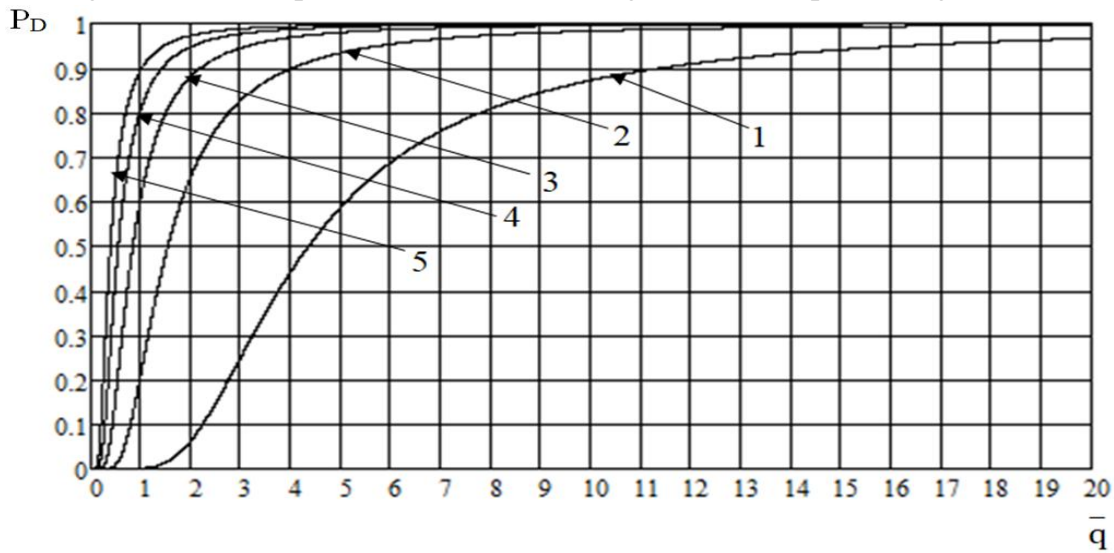

Figure 4: Multi-radar system detection characteristics: 1 - standalone radar; 2 - a multiradar system which consist of 2 radars; 3 - a multiradar system which consist of 3 radars; 4 - a multi-radar system which consist of 4 radars; 5 - a multiradar system which consist of 5 radars 
In the case of coherent signal summation and linear detection, the original statistics has a Rayleigh distribution, and in the case of quadratic detection, it has an exponential probability distribution. To construct detection curves in both cases, it is convenient to use a known expression for the probability of correct detection and false alarm (expression (3)):

$$
\mathrm{P}_{\mathrm{D}}=\mathrm{P}_{\mathrm{FA}} \frac{1}{\left(1+\overline{\mathrm{q}_{\text {exit }}^{2}}\right)},
$$

where $\mathrm{P}_{\mathrm{D}}$ - probability of correct detection;

$\mathrm{P}_{\mathrm{FA}}$ - probability of false alarm;

$\mathrm{q}_{\text {exit }}^{2}$ - signal-to-noise ratio at the input of the threshold device, which is determined depending on the features of the construction of a spatially coherent multi-radar system.

Figure 4 shows the case when all the same type of radar small base spatial-coherent multi-radar system uses the same signals so that they are in-phase summation on the air object and at the input of the receiving positions we get only one echo signal. The calculations for the operation of one autonomous radar and for the use of a multi-radar system with several radars for the probability of false alarm $\mathrm{P}_{\mathrm{FA}}=10^{-6}$ are given.

From the analysis of the detection characteristics shown on Figure 4, it is seen that the transition from a stand-alone radar (curve 1) to a combination of two or three radars (curves 2, 3) leads to a significant shift in the detection characteristics to the left. Increasing the number of radars combined coherently by more than three (curves 4,5 ) does not lead to a significant shift in the detection characteristics to the left compared to the detection characteristics when combining three radars (curve 2).

Figure 5 shows the dependence of the gain $\mathrm{K}(\mathrm{m})$ in the desired signal-to-noise ratio in each of the $\mathrm{m}$ radar from the multi-radar system relative to the autonomous radar to ensure quality detection of the air object in the spatially coherent multi-radar system $\left(\mathrm{P}_{\mathrm{D}}=0,5, \mathrm{P}_{\mathrm{FA}}=\right.$ $10^{-6}$ ) when the radar is coherently combined from the number of radars (m) combined.

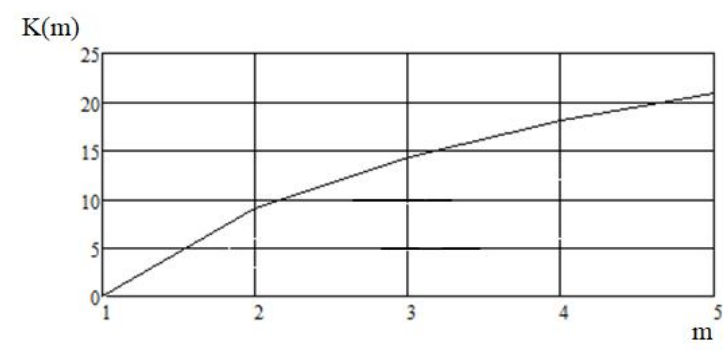

Figure 5 - The dependence of efficiency growth in relation signal / noise in a spatially coherent multi-radar system in the case where all radars use the same signals so that they are provided in-phase summation for an air object
From the analysis of the dependence of efficiency growth in relation signal / noise ratio, shown in Figure 5, it is seen that for the case when all the same type of radar small base spatial-coherent multi-radar system uses the same signals so that there is their in-phase summation on the air object with receiving at the input receiving positions only one echo signal, the gain is provided about $10 \mathrm{~dB}$ when combining two radars, and about $5 \mathrm{~dB}$ with the addition of a third radar, and increasing the gain by only $2.5 \mathrm{~dB}$ with the addition of a fourth radar and more radar also provide an increase in gain, but the rate of this growth is much lower, which worsens the practical significance and economic feasibility.

Therefore, summarizing the above, we can conclude that the greatest efficiency in the gain in the signal / noise ratio when combining the same spatially distributed radar into a coherent multi-radar system is observed in the transition from one autonomous radar to a multi-radar system with two-three radars.

A further increasing in the number of radars in all three cases is accompanied by a decreasing in the efficiency of each adding subsequent radar and it is almost impractical to use more than four radars in the multi-radar system with a maximum possible gain of about $2 \mathrm{~dB}$ with the addition of the last radar compared to almost $10 \mathrm{~dB}$ radar to multi-radar system with two radars.

The addition of the second radar showed the greatest efficiency in the gain in terms of signal / noise and all cases showed the optimal number of radars in the multi-radar system no more than four.

Thus, according to the efficiency-cost criterion, the most effective is the creation of a spatially coherent multi-radar system by combining two to four identical radars.

Thus, to improve the quality of detection of inconspicuous air objects, it is advisable to create a spatially coherent multi-radar system by combining two to four survey radars. In this case, the expected gain in the threshold signal / noise ratio can be up to $17 \mathrm{~dB}$. The use of more than four radars is impractical, as it does not lead to a significant increase in gain.

\section{CONCLUSION}

Thus, it can be argued that the greatest efficiency in the gain in terms of signal / noise showed the addition of second radar and the optimal number of radars in the multi-radar system is not more than four.According to the efficiency-cost criterion, the most effective is the creation of a spatially coherent multi-radar system by combining two to four identical radars.

Thus, to improve the quality of detection of inconspicuous air objects, it is advisable to create a spatially coherent multi-radar system by combining two 
to four survey radars. In this case, the expected gain in the threshold signal / noise ratio can be up to $18 \mathrm{~dB}$. The using of more than four radars is impractical, as it does not lead to a significant increase in gain. The current one was introduced.

The direction of further research is to consider the method of detecting an air object in the case of using in a multi-radar system with spatially spaced radar orthogonal mutually incoherent transmitting signal with their subsequent processing.

\section{REFERENCES}

1. M. Banasik, How to understand the Hybrid War, Securitologia, № 1, 2015. pp. 19-34. https://doi.org/10.5604/18984509.1184214

2. M. Banasik, Armed Forces As The Russian Federation's Strategic Tool, Journal on Baltic Security, № 5 (2), 2019, pp. 1-12. DOI: https://doi.org/10.2478/jobs-2019-0008.

3. V. Lishchenko, H. Khudov, V. Tiutiunnyk, V._Kuprii, F._Zots, and G._Misiyuk. The Method of Increasing the Detection Range of Unmanned Aerial Vehicles In Multiradar Systems Based on Surveillance Radars, in 2019 IEEE 39th International Conference on Electronics and Nanotechnology (ELNANO), 2019. DOI: https://doi.org/10.1109/ELNANO.2019.8783263.

4. I. Ruban et al., Segmentation of opticalelectronic images from on-board systems of remote sensing of the Earth by the artificial bee colony method, EEJET, № 2/9 (98), 2019, pp. 37-45. DOI: https://doi.org/10.15587/1729-4061.2019.161860.

5. P. Shchypanskyi, V. Savchenko, O. Martyniuk, and I. Kostiuk, Air Defense Planning from an Impact of a Group of Unmanned Aerial Vehicles based on Multi-Agent Modeling, IJETER, Vol. 8., № 4, 2020, pp. 7-12.

DOI: https://doi.org/10.30534/ijeter/2020/59842020

6. H. Khudov et al., Development of methods for determining the contours of objects for a complex structured color image based on the ant colony optimization algorithm, Eureka: Physics and Engineering, № 1, 2020, pp. 34-47. DOI: https://doi.org/10.21303/2461-4262.2020.001108.

7. I. Ruban et al., The Method for Selecting the Urban Infrastructure Objects Contours, in PIC S\&T, 2019, pp. 689-693.

DOI:https://doi.org/10.1109/PICST47496.2019.906 1267.

8. I. Ruban et al., Method for determining elements of urban infrastructure objects based on the results from air monitoring, EEJET, № 4/9 (100), 2019, pp. 52-61. https://doi.org/10.15587/1729-4061.2019.174576.

9. H. Khudov, A. Zvonko, S. Kovalevskyi, V. Lishchenko, and F. Zots. Method for the detection of smallsized air objects by observational radars, EEJET, № $2 / 9$ (92), 2018, pp. 61-68.

DOI:https://doi.org/10.15587/1729-4061.2018.126509

10. H. Khudov,

S. Kovalevskyi,

A. Irkha, V. Lishchenko, O. Serdiuk and F. Zots, The Proposals for Synchronization Positions of MIMO Radar System on the Basis of Surveillance Radars, in Intern. Scient.-Pract. Conf. Problems of Infocommunications. Science and Technology (PIC S\&T), 2019, pp. 547-551. DOI: https://doi.org/10.1109/PICST47496.2019.9061284.

11. H. Khudov, I. Khizhnyak, V. Koval, V. Maliuha, A. Zvonko, V. Yunda, V. Nagachevskyi, and V. Berezanskyi The Efficiency Estimation Method of Joint Search and Detection of Objects for Surveillance Technical Systems, IJETER, Vol. 8. № 3, 2020, pp. 813-819.

DOI: https://doi.org/10.30534/ijeter/2020/34832020

12. I. Ruban, H. Khudov, V. Lishchenko, A. Zvonko, S. Glukhov, I. Khizhnyak, V. Maliuha, Y. Polonskyi, R. Kushpeta, The Calculating Effectiveness Increasing of Detecting Air Objects by Combining Surveillance Radars into The Coherent System, IJETER, Vol. 8., № 4, 2020, pp. 1295-1301.

DOI:https://doi.org/10.30534/ijeter/2020/58842020.

13. V. Vasylyshyn Adaptive variant of the surrogate data technology for enhancing the effectiveness of signal spectral analysis using eigenstructure methods, Radioelectronics and Communications Systems, 2015, Vol. 58, pp. 116-126. DOI: https://doi.org/10.3103/S0735272715030036.

14. H. Khudov, I. Khizhnyak, I. Yuzova, O. Baranik, G. Semiv, S. Bondarenko, and O. Tytarenko. The Optimization Technique for Joint Discrete Search and Detection of Observation Objects, International Journal of Emerging Trends in Engineering Research, № 8(2), 2020, pp. 533-538. DOI:https://doi.org/10.30534/ijeter/2020/42822020.

15. H. Khudov, A. Fedorov, D. Holovniak, and G. Misiyuk. Improving the Efficiency of Radar Control of Airspace with the Multilateration System Use, in Intern. Scient.-Pract. Conf. Problems of Infocommunications. Science and Technology (PIC S\&T), 2018, pp. 680-684.

DOI: https://doi.org/10.1109/infocommst.2018.8632 141.

16. V. Lishchenko, V. Chaliy, H. Khudov, and A. Zvonko. Proposals for Improving of Air Surveillance Informativity in MIMO Radar Systems Based on Two-Dimensional Radars, in Intern. Scient.-Pract. Conf. Problems of Infocommunications. Science and Technology (PIC S\&T), 2018, pp. 153-156. DOI: https://doi.org/10.1109/infocommst.2018.8632052.

17. H. Khudov, A. Zvonko, I. Khizhnyak, V. Shulezko, V. Khlopiachyi, V. Chepurnyi, and I. Yuzova. The Synthesis of the Optimal Decision Rule for 
Detecting an Object in a Joint Search and Detection of Objects by the Criterion of Maximum Likelihood, IJETER, № 8(2), 2020, pp. 520-524.

DOI: https://doi.org/10.30534/ijeter/2020/40822020.

18. A. Farina, A. De Maio, and S. Haykin, The Impact of Cognition on Radar Technology, Stevenage: SciTech Publishing Inc., 2017.

19. H. Khudov, V. Lishchenko, H. Hyshko, Y. Polonskyi, I. Khizhnyak, B. Riabukha, The MIMO Surveillance Radars System with High Accuracy Finding 2D Coordinates, IJETER, № 8(5), 2020, pp. 2026-2030. DOI: https://doi.org/10.30534/ijeter/2020/91852020.

20. H. Khudov, S. Glukhov, O. Maistrenko, A. Fedorov, A. Andriienko, O. Koplik, The Method of ADS-B Receiver Systems Synchronization Using MLAT Technologies in the Course of Radar Control of Air Environment, IJETER, № 8(5), 2020, pp. 1946-1951. DOI:

https://doi.org/10.30534/ijeter/2020/78852020.

21. V. Lishchenko, T. Kalimulin, I. Khizhnyak, and H. Khudov, The method of the organization coordinated work for air surveillance in MIMO radar, Paper presented at the 2018 International Conference on Information and Telecommunication Technologies and Radio Electronics, UkrMiCo, 2018 - Proceeding.

DOI:https://doi.org/doi:10.1109/UkrMiCo43733.2018.904 7560. 